\title{
A BAYESIAN REVOLUTION IN SPECTRAL ANALYSIS
}

Published in "Bayesian Inference and Maximum Entropy Methods in Science and Engineering", Paris 2000, ed. A. Mohammad-Djafari, American Institute of Physics Proceedings,568, p.55\%, 2001

\author{
P. C. GREGORY \\ Dept. of Physics 83 Astronomy, University of British Columbia \\ 6224 Agricultural Rd., Vancouver, BC, Canada, V6T $1 Z 1^{\dagger}$
}

\begin{abstract}
The discrete Fourier transforms (DFT) is ubiquitous in spectral analysis as a result of the introduction of the Fast Fourier transform by Cooley and Tukey in 1965. In 1987, E. T. Jaynes derived the DFT using Bayesian Probability Theory and provided surprising new insights into its role in spectral analysis. From this new perspective the spectral resolution achievable is directly dependent on the signal to noise ratio and can be orders of magnitude better than that of a conventional Fourier power spectrum or periodogram. This was the starting point for an ongoing Bayesian revolution in spectral analysis which is reviewed in this paper, with examples taken from physics and astronomy. The revolution is based on a viewpoint of Bayesian Inference as extended logic.
\end{abstract}

\section{Introduction}

Science is all about identifying and understanding organized structures or patterns in nature. In this regard periodic patterns have proven especially important. Nowhere is this more evident than in the field of astronomy. Periodic phenomena allow us to determine fundamental properties like mass and distance, enable us to probe the interior of star through the new techniques of stellar seismology, detect new planets and discover exotic states of matter like neutron stars and black holes. Clearly any fundamental advance in our ability to detect periodic phenomena will have profound consequences in our ability to unlock nature's secrets. The purpose of this article is to describe such an advance and provide illustrations of its power through several examples in physics and astronomy.

This advance is based on a theory of extended logic called Bayesian probability theory (BPT) or Bayesian Inference. It is not the purpose of this paper to present the foundations of Bayesian inference. The reader is referred to the work of E. T. Jaynes $^{1}$. For a brief introduction to the subject see review articles by Loredo [19]

\footnotetext{
${ }^{\dagger}$ Email: gregory@physics.ubc.ca

${ }^{1}$ For example, see "Probability Theory: The Logic of Science", available at http://bayes.wustl.edu/.
} 
[20]. For a 4 page tutorial on how to do Bayesian inference see section 2 of Gregory and Loredo [11]. A more thorough pedagogical account is given in Devinder Sivia's introductory text [27].

For the purpose of this discussion scientific inference is defined as a method for assessing the plausibility of one or more competing models (hypotheses) to explain a class of phenomena, and estimating the model parameters. The hypothesis space can be either discrete or continuous as will be illustrated in the examples that follow. Bayesian inference differs from conventional frequentist statistical inference in the definition of probability that is used. ${ }^{2}$ This important difference allows Bayesian inference to bridge in a continuous way to deductive logic. Deductive logic is based on axiomatic knowledge. However, in scientific inference our reasoning is based on incomplete information. There are always more experiments that can be done to test the predictions of a particular theory or hypothesis and a variety of errors can be introduced in any measurement operation. Our conclusions are at best probabilities. The role of extended logic is to provide a well defined procedure for encoding our current state of knowledge, about some hypothesis space of interest, into a probability distribution. It is common practice to represent a probability by a real number in the range 0 to 1 , with the end points 0 and 1 standing for absolutely false and absolutely true, respectively. In this context deductive logic can be shown to be a special case of BPT in the idealized limit of complete information where the probability is either 0 or 1 .

The power of present day computers readily permits testing of the efficacy of different approaches to scientific inference with well understood simulated data sets, but ultimately we are interested in their success when applied to real scientific problems. The rest of this paper will focus on recent demonstrations of the capabilities of Bayesian inference in the arena of spectral analysis starting with E. T. Jaynes's new insights.

\section{New Insights on the Periodogram from Probability Theory}

Arthur Schuster introduced the periodogram in 1905, as a means for detecting a periodicity and estimating its frequency. If the data are evenly spaced, the periodogram is determined by the discrete Fourier transforms (DFT), thus justifying the use of the DFT for such detection and measurement problems. In 1965 [8] Cooley and Tukey introduced the Fast discrete Fourier transform (FFT).

The Schuster periodogram was introduced largely for intuitive reasons, but in 1987 Jaynes provided a formal justification by applying the principles of Bayesian inference to the following [17]. Suppose we are analyzing data consisting of samples of a continuous function contaminated with additive independent Gaussian noise

\footnotetext{
${ }^{2}$ In frequentist statistical inference the probability, $p(A)=$ long-run relative frequency with which outcome A occurs in identical repeats of an experiment. " $A$ " restricted to propositions about random variables.

In Bayesian inference, $p(H \mid D, I)=$ a real number measure of the plausibility of a proposition/hypothesis $H$, given the truth of the information represented by propositions $D$ (new data) and $I$ (prior information). " $H$ " can be any logical proposition, i.e. not restricted to propositions about random variables.
} 
with a variance of $\sigma^{2}$. Jaynes showed that, presuming the possible periodic signal is sinusoidal (but with unknown amplitude, frequency, and phase), the Schuster periodogram exhausts all the information in the data relevant to assessing the possibility that a signal is present, and to estimating the frequency and amplitude of such a signal. The periodogram is essentially the squared magnitude of the DFT and can be defined as

$$
\begin{aligned}
\text { periodogram }=C\left(f_{n}\right) & =\frac{1}{N}\left|\sum_{k=1}^{N} d_{k} e^{i 2 \pi n \Delta f k \Delta t}\right|^{2} \\
& =\frac{1}{N}|F F T|^{2}
\end{aligned}
$$

In a FFT the frequency interval, $\Delta f=1 / T$, where $T$ is the duration of the data set consisting of $N$ samples with a sample interval $\Delta t$. The quantity $C\left(f_{n}\right)$ is indeed fundamental to spectral analysis but not because it is itself a satisfactory spectrum estimator. Jaynes showed that the probability for the frequency of a periodic sinusoidal signal is given approximately ${ }^{3}$ by

$$
p\left(f_{n} \mid D, I\right) \propto \exp \left\{\frac{C\left(f_{n}\right)}{\sigma^{2}}\right\}
$$

Thus the proper algorithm to convert $C\left(f_{n}\right)$ to $p\left(f_{n} \mid D, I\right)$ involves first dividing $C\left(f_{n}\right)$ by the noise variance and then exponentiating. This naturally suppresses spurious ripples at the base of the periodogram, usually accomplished with linear smoothing; but does it by attenuation rather than smearing, and therefore does not sacrifice any precision. The Bayesian nonlinear processing of $C\left(f_{n}\right)$ also yields, when the data give evidence for them, arbitrarily sharp spectral peaks. Since the peak in $p\left(f_{n} \mid D, I\right)$ can be much sharper than the peak in $C\left(f_{n}\right)$, it is necessary to zero pad the FFT to obtain a sufficient density of points in $C\left(f_{n}\right)$ for use in equation (2) to accurately define a peak in $p\left(f_{n} \mid D I\right)$.

Figure 1 provides a demonstration of these properties for a simulated data set consisting of a single sine wave plus additive Gaussian noise given by equation (3).

$$
y=A \cos 2 \pi f t+\text { Gaussian noise (mean }=0, \sigma=1)
$$

where $A=1, f=0.1 \mathrm{~Hz}$. The upper panel shows 64 simulated data points computed from equation (3), with one $\sigma$ error bars. The middle panel is the Fourier power spectral density or periodogram, computed for this data according equation (1). ${ }^{4}$ The sinusoidal signal is clearly indicated by the prominent peak. The signal to noise ratio $(\mathrm{S} / \mathrm{N})$, defined as the ratio of the RMS signal amplitude to the noise $\sigma$, was 0.7 in the above simulation. If we repeated the simulation with a larger $\mathrm{S} / \mathrm{N}$ ratio, the main peak would increase in relation to the noise peaks and

\footnotetext{
${ }^{3}$ Bretthorst [6] derives the exact result for the more general case of nonuniformily sampled data which involves an analogous nonlinear transformation of the Lomb-Scargle periodogram [18] [23] [24]. Bretthorst [7] also shows how to generalize the Lomb-Scargle periodogram for the case of a nonstationary sinusoid.

${ }^{4}$ The 64 points were zero padded to provide a total of 512 points for the FFT.
} 
we would start to notice side lobes emerging associated with the finite duration of the data set (rectangular window function). However, a well known property of the periodogram is that the width of any spectral peak depends only on the duration of the data set and not on the signal to noise level. Various methods have been used to determine the accuracy to which the peak frequency can be determined, but, as we see below, the Bayesian posterior probability for the signal frequency provides this information directly.

The lower panel of figure 1 shows the Bayesian probability density for the period of the signal, derived from equation (2). As the figure demonstrates the spurious noise feature are suppressed and the width of the spectral peak is much narrower than the peak in the periodogram. In a Bayesian analysis the width of spectral peak, which reflects the accuracy of the frequency estimate, is determined by the duration of the data, the $\mathrm{S} / \mathrm{N}$ ratio and the number of data points. More precisely the standard deviation of the spectral peak, $\delta f$, for a $\mathrm{S} / \mathrm{N}>1$, is given by

$$
\delta f \approx\left(1.6 \frac{\mathrm{S}}{\mathrm{N}} T \sqrt{N}\right)^{-1} \mathrm{~Hz}
$$

where $T=$ the data duration in s, $N=$ the number of data points in $T$.

Equation (2) assumes that the noise variance is a known quantity. In some situations the noise is not well understood, i.e. our state of knowledge is less certain. Even if the measurement apparatus noise is well understood, the data may contain a greater complexity of phenomena than the current signal model incorporates. In such cases equation (2) is no longer relevant, but again, Bayesian inference can readily handle this situation [6] by treating the noise variance as an unknown quantity, or by making use of whatever prior information we have about the noise. In this case the analysis treats everything that cannot be explained by the signal model as noise, leading to the most conservative estimates.

A corollary of Jaynes's analysis is that for any other problem (e.g., nonsinusoidal light curve, non Gaussian noise, or nonuniform sampling) use of the FFT is not optimal; more information can be extracted from the data if we use more sophisticated statistics. Jaynes made this point himself, and it has been amply demonstrated in the work of Bretthorst [1], who has applied similar methods to signal detection and estimation problems with non sinusoidal models with Gaussian noise probabilities. In the following sections we will consider two general classes of spectral problems: (a) those for which we have strong prior information of the signal model, and (b) those for which we have no specific prior information about the signal.

\section{Spectral Analysis with Strong Prior Information of the Signal Model}

Larry Bretthorst [1] [2] [3] [4] [5] extended Jaynes's work to more complex signal models with additive Gaussian noise and revolutionized the analysis of Nuclear Magnetic Resonance (NMR) signals. In NMR free-induction decay, the signal consists of a sum of exponentially decaying sinusoids of different frequency and decay rate. The upper two panels of figure 2 illustrate the quadrature channel measurements in a NMR free-induction decay experiment. In this example the S/N 

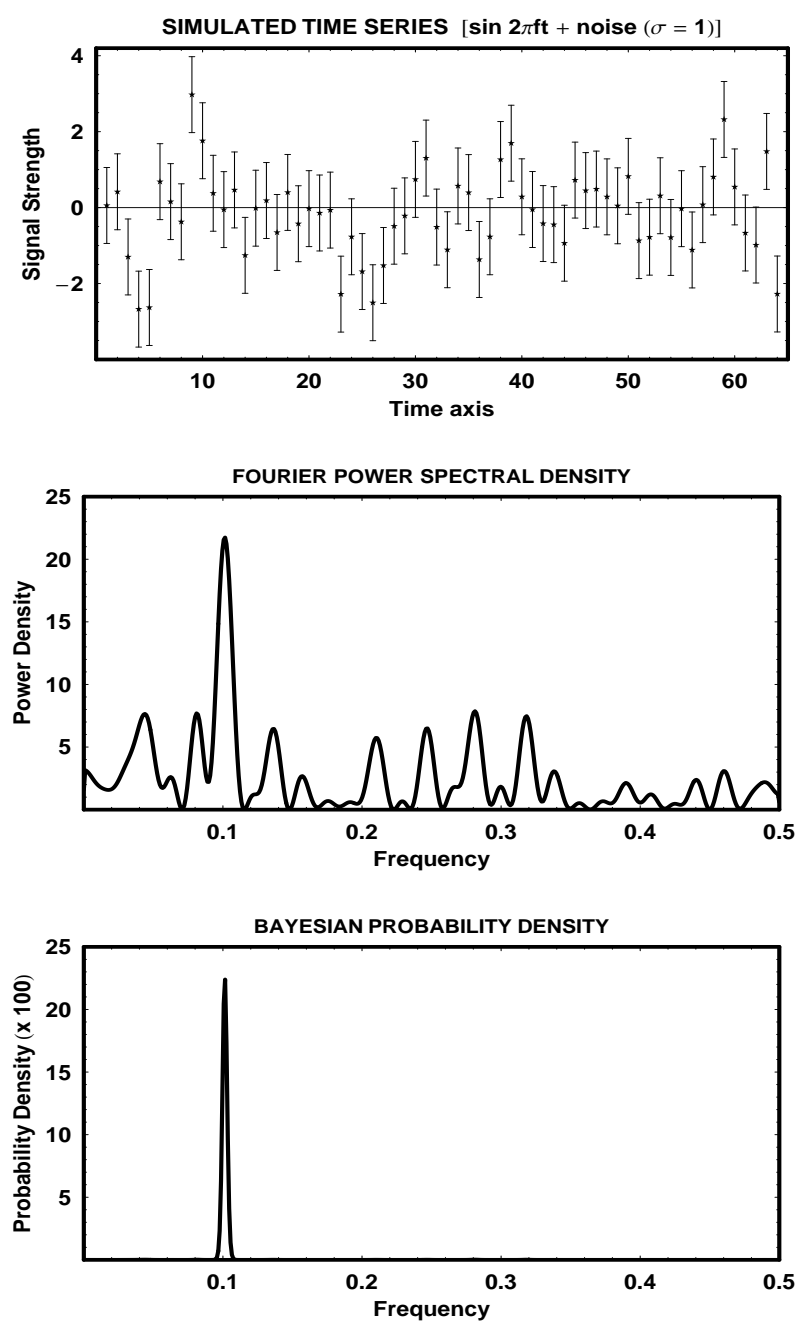

Figure 1. Comparison of conventional (middle panel) and Bayesian analysis (lower panel) of a simulated time series (upper panel).

ratio is very high. The middle panel illustrates the conventional absorption spectrum based on a FFT of the data, which shows three obvious spectral peaks with an indication of further structure in the peaks. The lower panel illustrates Bretthorst's Bayesian analysis of this NMR data which clearly isolates six separate peaks. The resolution is so good that the 6 peaks appear as delta functions in this figure. A similar improvement was obtained in the estimation of the decay rates. The Bayesian analysis provides much more reliable and informative results when prior knowledge of the shape of the signal and noise statistics are incorporated. 

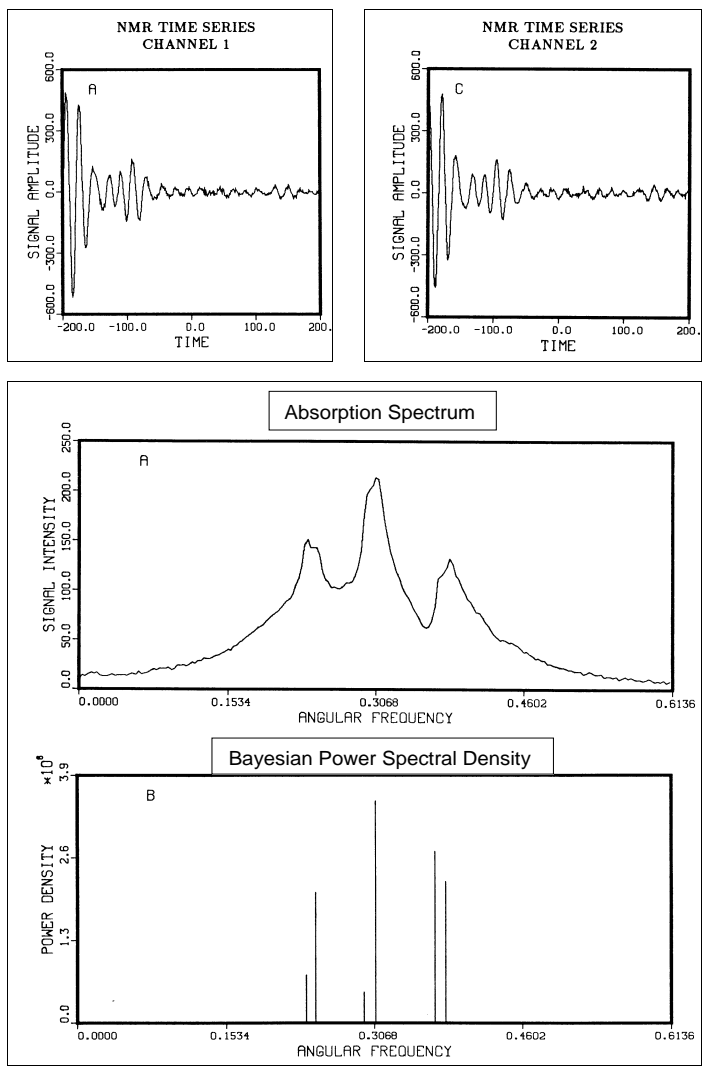

Figure 2. Comparison of conventional analysis (middle panel) and Bayesian analysis (lower panel) of the two channel NMR time series (upper two panels). (Ref: G. L. Bretthorst, "Bayesian Spectrum Analysis and Parameter Estimation", Springer Verlag, 1988)

Varian Corporation now offers an expert analysis package with their new NMR machines based on Bretthorst's Bayesian algorithm. His work is very relevant to many current problems in science, e.g., the detection of extrasolar planets and stellar seismology.

\section{Spectral Analysis with No Specific Prior Information of the Signal}

In this case we are addressing the detection and measurement of a periodic signal in a time series when we have no specific prior knowledge of the existence of such a signal or of its characteristics, including its shape. For example, an extraterrestrial civilization might be transmitting a repeating pattern of information either 
intentionally or unintentionally. What scheme could we use to optimally detect such a signal after we have made our best guess at a suitable wavelength of observation? Bayesian inference provides a well defined procedure for solving any inference problem including questions of this kind. However, to proceed with the calculation it is necessary to assume a model or family of models which is capable of approximating a periodic signal of arbitrary shape. A very useful Bayesian solution to the problem of detecting a signal of unknown shape was worked out by the author in collaboration with Tom Loredo of Cornell University [11] [12] [13] in the case of event arrival time data.

We used a family of histogram (piecewise constant) signal models, with each member of the family having a different number of bins $m$, with $m$ ranging from 2 to some upper limit, typically 12 . The Bayesian calculation automatically incorporates a quantified Occam's penalty, penalizing models with larger number of bins for their greater complexity. The calculation thus balances model simplicity with goodness-of-fit, allowing us to determine both whether there is evidence for a periodic signal, and the optimum number of bins for describing the structure in the data. A remarkable feature of this particular signal model is that it leads to a computationally tractable algorithm, because many of the required calculations can be performed analytically. Further research is underway to investigate computationally tractable ways of incorporating additional desirable features into the signal model, such as, variable bin widths to allow for a reduction in the number of bins needed to describe certain types of signals.

The Gregory-Loredo (GL) algorithm was motivated by the problem of detecting astronomical X-ray pulsars where the data consists of the arrival times of individual X-ray photons, some or all of which are background events, and the appropriate sampling distribution is the Poisson distribution. More recently the author has published [14] a Bayesian solution to the unknown signal detection problem for the Gaussian noise case.

The solution in the Poisson case yields a result that is intuitively very satisfying. The probability for the family of periodic models can be shown to be approximately inversely proportional to the entropy [11] of any significant organized periodic structure found in the search parameter space. What structure is significant is determined through built in quantified Occam's penalties in the calculation. Of course structure with a high degree of organization corresponds to a state of low entropy. In the absence of knowledge about the shape of the signal the method identifies the most organized significant periodic structure in the model parameter space.

Some of the capabilities of the GL method are illustrated in the following two examples, one taken from X-ray astronomy and the other from radio astronomy.

\subsection{X-RAY ASTRONOMY EXAMPLE}

In 1984 Seward et al. [25] discovered a $50 \mathrm{~ms}$ X-ray pulsar at the center of a previously known radio supernova remnant, SNR 0540-693, located in the Large Magellanic Cloud. The initial detection of X-ray pulsations was from an FFT periodogram analysis of the data obtained from the Einstein Observatory. The 


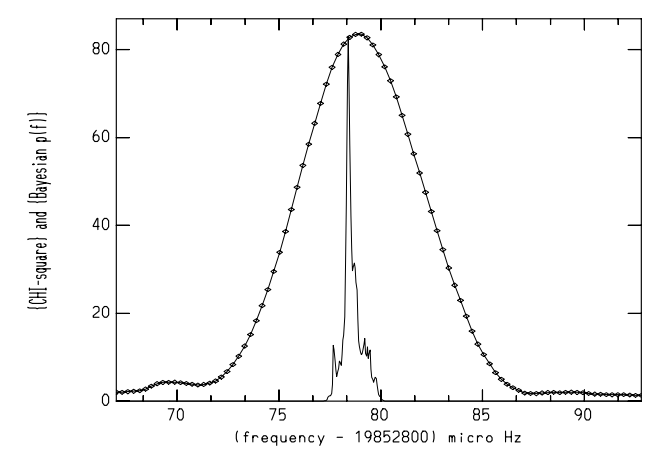

Figure 3. Close up of largest frequency peak comparing the Gregory-Loredo probability density for $f$ (solid curve) to the Epoch Folding $\left\langle\chi^{2}\right\rangle_{\phi}$ statistic (diamonds). The $\left\langle\chi^{2}\right\rangle_{\phi}$ statistic versus trial frequency results from epoch folding analysis using $m=5$ bins (Ref: Gregory and Loredo, Ap. J., 473, 1059)

true pulsar signal turned out to be the second highest peak in the initial FFT. Confidence in the reality of the signal was established from FFT runs on other data sets. The pulsar was re-observed with the ROSAT Observatory by Seward and colleagues, but this time an FFT search failed to detect the pulsar. In reference [13] we used the GL method on a sample ROSAT data set of 3305 photons provided by F. Seward. The data spanned an interval of 116,341 s and contained many gaps.

In the first instance we incorporated the prior information on the period, period derivative and their uncertainties, obtained from the earlier detection with the Einstein Observatory data. The GL method provides a calculation of the global odds ratio defined as the ratio of the probability for the family of periodic models to the probability of a constant rate model, regardless of the exact shape, period and phase of the signal. The resulting odds ratio of $2.6 \times 10^{11}$ indicates near certainty in the presence of a periodic signal. In the second instance we ignored the results of the previous detection and assumed a prior period search range extending from the rotational breakup period of a neutron star $(\approx 1.5 \mathrm{~ms})$, to half the duration of the data. This gave an odds ratio of $=4.5 \times 10^{5}$. This is greatly reduced due to the much larger Occam penalty associated with not knowing the period as well. But this still provided overwhelming evidence for the presence of a periodic signal, despite the fact that it was undetected by FFT techniques.

In their paper Seward et al. [25] used another method commonly employed in X-ray astronomy, called Epoch Folding, to obtain the pulsar light curve and a best period. Epoch Folding involves dividing the trial period into $m$ bins (typically 5 ) and binning the data modulo the trial period for a given trial phase. The $\chi^{2}$ statistic is used to decide at some significance level, whether a constant model can be rejected, and thus indirectly infer the presence of a periodic signal. In Seward et al. [25], their period uncertainty was estimated from the half-width of the $\chi^{2}$ peak, which is sometimes used as a naive estimate of the accuracy of the frequency estimate. Figure 3 shows a comparison of the largest frequency peak comparing 
the GL marginal probability density for $f$ to the Epoch Folding $\left\langle\chi^{2}\right\rangle_{\phi}$ statistic. The width of the GL marginal probability density for $f$ is more than an order of magnitude smaller.

\subsection{RADIO ASTRONOMY EXAMPLE}

The author has recently generalized the Gregory-Loredo Algorithm to the Gaussian noise case [14]. Application of the method to a radio astronomy data set has resulted in the discovery of a new periodic phenomena [14] [15] in the X-ray and radio emitting binary, $\mathrm{LS} \mathrm{I}+61^{\circ} 303$. $\mathrm{LS} \mathrm{I}+61^{\circ} 303$ is a remarkable $10^{\text {th }}$ magnitude binary star [9] [16] that exhibits periodic radio outbursts every 26.5 days [28], which is the binary orbital period. The radio, infrared, optical, X-ray and $\gamma$-ray data indicate that the binary consists of a rapidly rotating massive young star, called a Be star, together with a neutron star in an eccentric orbit.

The Be star exhibits a dense equatorial wind and the periodic radio outbursts are thought to arise from variations in wind accretion by the neutron star in its eccentric orbit. Some of the energy associated with the accretion process is liberated in the form of outbursts of radio emission. One puzzling feature of the outbursts has been the variability of the orbital phase of the outburst maxima, which can range over 180 degrees of phase. In addition the strength of the outburst peaks was known to vary on time scales of approximately 4 years [10] [21].

Armed with over twenty years of data Gregory [14] and Gregory et al. [15] applied Bayesian inference to assess a variety of hypotheses to explain the outburst timing residuals and peak flux density variations. The results for both the outburst peak flux density and timing residuals demonstrated a clear 1580 day periodic modulation in both quantities. The periodic modulation model was found to be $\sim 3 \times 10^{3}$ times more probable than the sum of the probabilities of three competing nonperiodic models.

Figure 4 shows the data and results from the timing residual analysis. The upper panel shows the radio outburst peak timing residuals ${ }^{5}$. The abscissa is the time interval in days from the peak of the first outburst in 1977. Very sparsely sampled measurements were obtained from the initial discovery in 1977 until 1992. However, beginning in January 1994 Ray et al. [22], detailed monitoring was performed (several times a day) with the National Radio Astronomy Observatory Green Bank Interferometer. With such sparsely sampled data the eye is unable to pick out any obvious periodicity. The middle panel shows the Bayesian marginal probability density for the modulation period. The single well defined peak provides clear evidence for a periodicity of approximately 1580 days. The lower panel shows the Bayesian estimate of the shape of the timing residual variations and the data, repeated for two cycles of phase. The two solid curves are the Bayesian computed mean shape \pm 1 standard deviation. The phase of the data was computed using the most probable modulation period of 1580 days. A similar analysis of the peak flux density data [14] yields the same modulation period within the errors.

\footnotetext{
${ }^{5}$ The timing residuals depend on the assumed orbital period which is not accurately known independent of the radio data.
} 

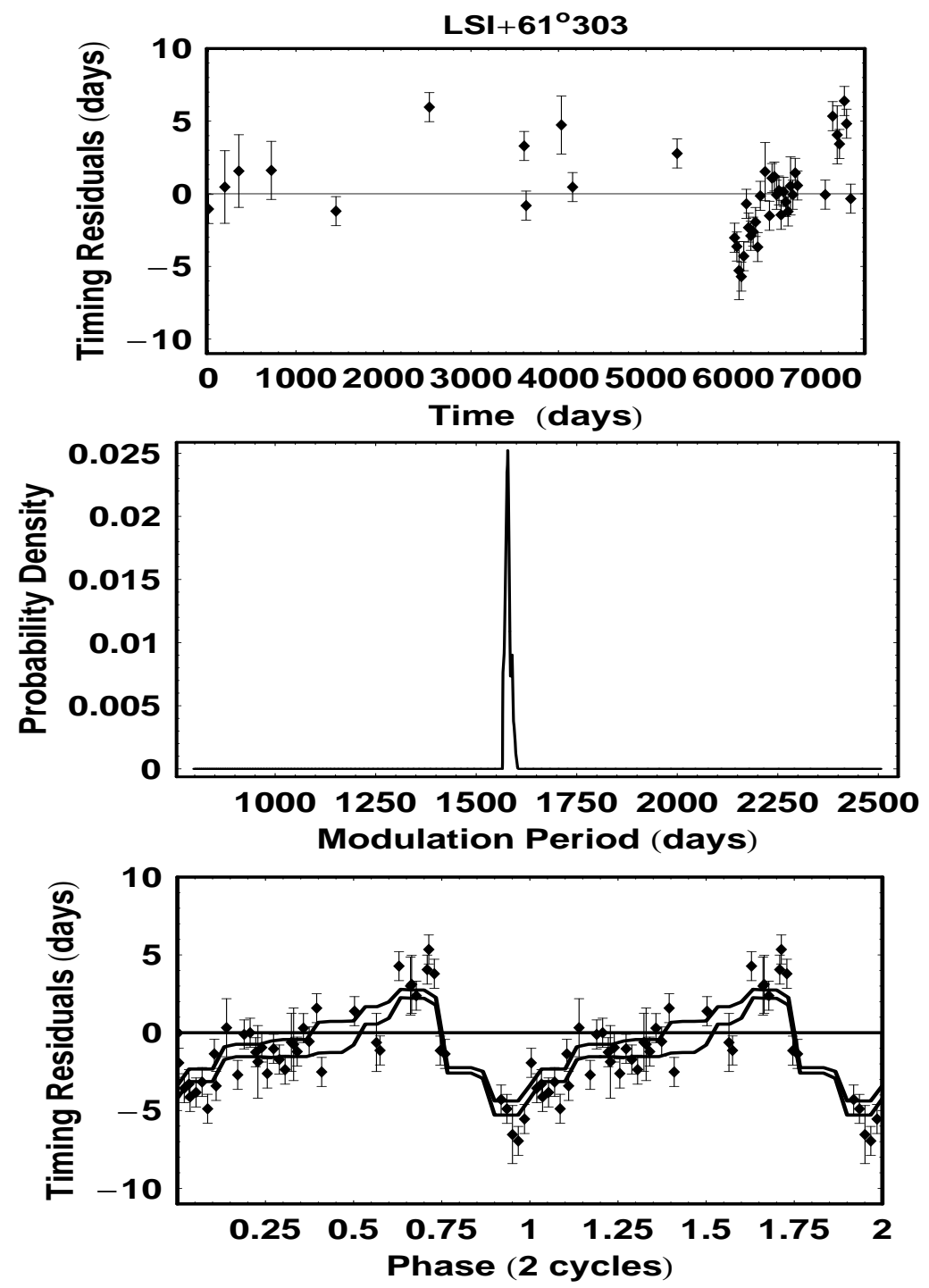

Figure 4. a) The upper panel shows the outburst timing residuals, b) The middle panel shows the probability for the modulation period of LS I $+61^{\circ} 303$ timing residuals. c) In the lower panel the two solid curves are the estimated mean light curve, \pm 1 standard deviation, of the timing residual periodic modulation, repeated for two cycles of phase. The phase of the raw data was computed using the most probable modulation period of 1580 days.

Subsequent monitoring of the binary has confirmed and refined the orbital and modulation period. Figure 5 shows a comparison of the predicted outburst timing residuals with the data versus time. The solid curves show the estimated mean 


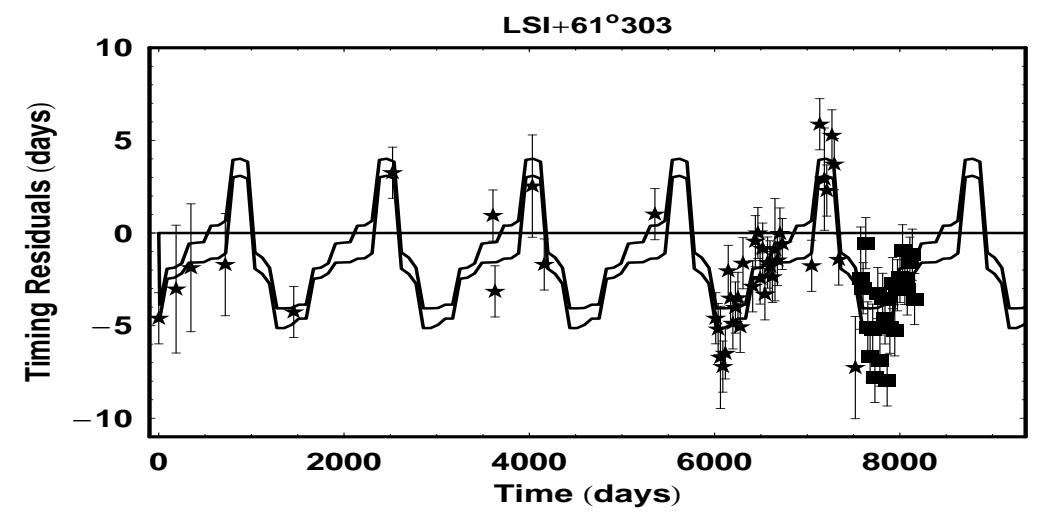

Figure 5. Comparison of the predicted outburst timing residuals with the data versus time. The solid curves show the estimated mean light curve, \pm 1 standard deviation. The new data is indicated by a shaded box.

light curve, \pm 1 standard deviation. The new data, indicated by a shaded box, nicely confirms the periodic modulation model. This discovery has contributed significantly to our understanding of Be star winds.

\section{Conclusions}

The aim of this review has been to draw the attention of the larger scientific community to the power of Bayesian inference through recent examples of its use in spectral analysis. For scientific data with a high signal to noise ratio, a Bayesian analysis can frequently yield many orders of magnitude improvement in model parameter estimation. It also provides a powerful way of assessing competing theories at the forefront of science by quantifying Occam's razor and provides a means for incorporating the effects of suspected systematic errors. For some problems a Bayesian analysis may lead to a familiar statistic but even in this situation it often yields powerful new insights concerning the interpretation and generalization of the statistic. But most importantly, as a mathematical theory of extended logic it provides a well defined procedure for answering any scientific inference question for a given state of knowledge.

This research was supported in part by a grant from the Canadian Natural Sciences and Engineering Research Council at the University of British Columbia.

\section{References}

1. Bretthorst, G.L. (1988) Bayesian Spectrum Analysis and Parameter Estimation, SpringerVerlag, N.Y.

2. Bretthorst, G.L. (1990) Bayesian Analysis. I. Parameter Estimation Using Quadrature NMR Models, Journal of Magnetic Resonance 88, 533-551.

3. Bretthorst, G.L. (1990) Bayesian Analysis. II. Signal Detection and Model Selection, Journal of Magnetic Resonance 88, 552-570.

4. Bretthorst, G.L. (1990) Bayesian Analysis. III. Applications to NMR Signal Detection, Model 
Selection, and Parameter Estimation, Journal of Magnetic Resonance 88, 571-595.

5. Bretthorst, G.L. (1991) Bayesian Analysis. IV. Noise and Computing Time Considerations, Journal of Magnetic Resonance 93, 369-394.

6. Bretthorst, G.L. (2000) Nonuniform Sampling: Bandwidth and Aliasing, to appear in Bayesian Inference and Maximum Entropy Methods, ed. G. Erickson, Kluwer Academic Publishers.

7. Bretthorst, G.L. (2001) Generalizing the Lomb-Scargle Periodogram, to appear in Bayesian Inference and Maximum Entropy Methods in Science and Engineering, Paris 2000, ed. Ali Mohammad-Djafari, American Institute of Physics Proceedings.

8. Cooley, J. W., and Tukey. J. W. (1965) An Algorithm for the Machine Calculation of Complex Fourier Series, Mathematics of Computing, 19, 297-301.

9. Gregory, P. C., and Taylor, A. R. 1978 New Highly Variable Radio Source, Possible Counterpart of gamma-Ray Source CG 135+1, Nature, 272, 704-706.

10. Gregory, P.C., Xu, H.J., Backhouse, C.J. and Reid, A. (1989) Four-Year Modulation of Periodic Radio Outbursts from LS I+61 303 , Astrophysical Journal,339, 1054-58.

11. Gregory, P. C., and Loredo, T. J. (1992) A New Method for the Detection of a Periodic Signal of Unknown Shape and Period. Astrophysical Journal, 398, 146-168.

12. Gregory, P. C., and Loredo, T. J. (1993) A Bayesian Method for the Detection of Unknown Periodic and Non periodic Signals in Binned Time Series, in Maximum Entropy and Bayesian Methods, Paris, ed. Mohammad-Djafari and G. Demoment, (Kluwer academic press, Dordrecht), 225-232.

13. Gregory, P. C., and Loredo, T. J. (1996) Bayesian Periodic Signal Detection: Analysis of ROSAT Observations of PSR 0540-693, Astrophysical Journal, 473, 1059-1066.

14. Gregory, P. C. (1999) Bayesian Periodic Signal Detection: Analysis of 20 Years of Radio Flux Measurements of LS I+61 ${ }^{\circ} 303$, Astrophysical Journal, 520, p. 361-375.

15. Gregory, P. C., Peracaula, M. and Taylor, A.R. (1999) Bayesian Periodic Signal Detection: Discovery of Periodic Phase Modulation in LS I+61 303 Radio Outbursts, Astrophysical Journal, 520, p. 376-390.

16. Hutchings, J. B., and Crampton, D. (1981), Spectroscopy of the unique degenerate binary star LS I+61 ${ }^{\circ} 303, P A S P, \mathbf{9 3}, 486-489$.

17. Jaynes, E.T. (1987) Bayesian Spectrum \& Chirp Analysis, in Maximum Entropy and Bayesian Spectral Analysis and Estimation Problems, ed. C.R. Smith and G.L. Erickson, D. Reidel, Dordrecht, 1-37.

18. Lomb, N. R. (1976) Least Squares Frequency Analysis of Unevenly Spaced Data, Astrophysical ans Space Sciences, 39, 447-462.

19. Loredo, T. J. (1990) From Laplace to Supernova SN 1987A: Bayesian Inference in Astrophysics, Maximum Entropy and Bayesian Methods, Dartsmouth, ed. P. Fougère, Dordrecht: Kluwer Academic Publishers, 81-142.

20. Loredo, T. J. (1992) The Promise of Bayesian Inference for Astrophysics, Statistical Challenges in Modern Astronomy, eds. E. D. Feigelson and G. B. Babu, New York: Springer-Verlag, 257-297.

21. Paredes, J. M., Estelle, R., Ruis, A. (1990) Observation at $3.6 \mathrm{~cm}$ wavelength of the radio light curve of LS I+61 303 , Astronomy and Astrophysics, 232, 377-380.

22. Ray, P. S., Foster, R.S., Waltman, E. B. et al. (1997) Long Term Monitoring of LS I+61 303 at 2.25 and $8.3 \mathrm{GHz}$, Astrophysical Journal, 491, 381-387.

23. Scargle, J. D. (1982) Studies in Astronomical Time Series Analysis II. Statistical Aspects of Spectral Analysis of Unevenly Sampled Data, Astrophysical Journal, 263, 835-853.

24. Scargle, J. D. (1989) Studies in Astronomical Time Series Analysis III. Autocorrelation and Cross-correlation Functions of Unevenly Sampled Data, Astrophysical Journal, 343, 874-887.

25. Seward, F. D., Harnden, F. R., and Helfand, D. J. (1984) Discovery of a 50 millisecond pulsar in the Large Magellanic Cloud, Astrophysical Journal Letters, 287, L19-22.

26. Schuster, A. (1905) The Periodogram and its Optical Analogy, Proceedings of the Royal Society of London, 77, 136-140.

27. Sivia, D. S. (1996) Data Analysis: A Bayesian Tutorial, Oxford University Press.

28. Taylor, A.R. and Gregory, P.C. (1982) Periodic Radio Emission From LS I+61 ${ }^{\circ} 303$, As- $^{-}$ trophysical Journal, 255, 210-216. 